\title{
Multipulse Chaotic Dynamics for a Laminated Composite Piezoelectric Plate
}

\author{
J. H. Zhang and W. Zhang \\ College of Mechanical Engineering, Beijing University of Technology, Beijing 1000124, China \\ Correspondence should be addressed to J. H. Zhang, zjh@bjut.edu.cn
}

Received 17 August 2010; Revised 9 February 2011; Accepted 16 February 2011

Academic Editor: E. E. N. Macau

Copyright (C) 2011 J. H. Zhang and W. Zhang. This is an open access article distributed under the Creative Commons Attribution License, which permits unrestricted use, distribution, and reproduction in any medium, provided the original work is properly cited.

We investigate the global bifurcations and multipulse chaotic dynamics of a simply supported laminated composite piezoelectric rectangular thin plate under combined parametric and transverse excitations. We analyze directly the nonautonomous governing equations of motion for the laminated composite piezoelectric rectangular thin plate. The results obtained here indicate that the multipulse chaotic motions can occur in the laminated composite piezoelectric rectangular thin plate. Numerical simulations including the phase portraits and Lyapunov exponents are used to analyze the complex nonlinear dynamic behaviors of the laminated composite piezoelectric rectangular thin plate.

\section{Introduction}

Piezoelectric materials can be used as the actuators and sensors in engineering structures [1]. With the increased use of composite laminated piezoelectric plates in engineering structures, for example, in airplane and launch vehicles, research works and development on the responses of laminated composite piezoelectric plates have experienced tremendous growth in the last two decades. For instance, Ye and Tzou [2] developed a new piezoelectric composite finite element and gave a comparison between finite element solutions of a laminated composite piezoelectric plate and experimental data. Shen [3] analyzed nonlinear bending of a simply supported, shear deformable cross-ply laminated plate with piezoelectric actuators and subjected to a transverse uniform or sinusoidal load combined with electrical loads and thermal environments based on higher-order shear deformation plate theory and perturbation technique. Recently, Zhang et al. [4] established the nonlinear governing equations of motion for a simply supported laminated composite piezoelectric rectangular plate under combined parametric and transverse excitations and studied the periodic and 
chaotic dynamics in the case of one-to-two internal resonance. The linear and geometrically nonlinear forced vibrations of laminated composite plates with piezoelectric materials were studied through numerical approach by Tanveer and Singh [5]. Torres and Mendonca [6] developed a formulation for laminated plates with extensional distributed piezoelectric sensors and the analytical solutions for simply supported square laminates with piezoelectric layers.

Laminated composite plates with piezoelectric materials can undergo large oscillating deformation, which leads to nonlinear oscillations of plates. However, little research deals with the complex nonlinear dynamics of laminated composite piezoelectric plates, such as the bifurcations and multipulse chaotic dynamics. We investigate chaotic phenomena in such systems in order that we can control the system through the piezoelectric change. With the development of the theories of nonlinear dynamics and chaos, prediction, understanding, and control become possible for more complicated nonlinear phenomena in laminated composite piezoelectric plates. Kovacic and Wiggins [7] developed a new global perturbation method which may be used to detect the Shilnikov-type single-pulse homoclinic and heteroclinic orbits of four-dimensional autonomous ordinary differential equations. Camassa et al. [8] proposed an extended Melnikov method to study the multipulse jumping of homoclinic and heteroclinic orbits in a class of perturbed Hamiltonian systems. Zhang et al. [9] improved the extended Melnikov method given by Camassa et al. [8] and employed it to study the multipulse Shilnikov-type chaotic dynamics for a nonautonomous buckled rectangular thin plate. Zhang et al. [10] presented an extended Melnikov method to nonautonomous nonlinear dynamical systems in mixed coordinates. The multipulse chaotic dynamics of a simply supported laminated composite piezoelectric rectangular thin plate under the combination of the parametric and transverse excitations was investigated by the proposed method.

However, in paper [10], the normal form theory is used to simplify the equations of the laminated composite piezoelectric plate. In this paper, the multipulse chaotic dynamics of the simply supported laminated composite piezoelectric rectangular plate under combined parametric and transverse excitations is investigated by using the extended Melnikov method improved in paper [9]. The nonlinear terms, which were missing through simplification by normal form theory in paper [10], are retained in this paper and added small positive parameter.

\section{Analysis on Multipulse Chaotic Dynamics of the Plate}

In this section, we investigate the multipulse chaotic dynamics for the simply supported laminated composite piezoelectric rectangular thin plate under combined parametric and transverse excitations. The two-degree-of-freedom governing equation of motion for the plate in dimensionless nonautonomous nonlinear system is shown in (2.1), and the details about the equation can be seen in paper [4]:

$$
\begin{aligned}
\ddot{w}_{1}+ & \mu_{1} \dot{w}_{1}+\omega_{1}^{2} w_{1}+\left(f_{1} \cos \Omega_{1} t+f_{2} \cos \Omega_{2} t+f_{3} \cos \Omega_{4} t\right) w_{1} \\
& +\alpha_{1} w_{1} w_{2}^{2}+\alpha_{2} w_{1}^{2} w_{2}+\alpha_{3} w_{1}^{3}+\alpha_{4} w_{2}^{3}=F_{1} \cos \Omega_{3} t \\
\ddot{w}_{2}+ & \mu_{2} \dot{w}_{2}+\omega_{2}^{2} w_{2}+\left(f_{4} \cos \Omega_{1} t+f_{5} \cos \Omega_{2} t+f_{6} \cos \Omega_{4} t\right) w_{2} \\
& +\beta_{1} w_{1}^{2} w_{2}+\beta_{2} w_{1} w_{2}^{2}+\beta_{3} w_{2}^{3}+\beta_{4} w_{1}^{3}=F_{2} \cos \Omega_{3} t
\end{aligned}
$$


where $\alpha_{i}$ and $\beta_{i}$ (i=1-4) are nondimensional coefficients. In (2.1), the coefficients for $\omega_{1}$, $\omega_{2}, F_{1}, F_{2}, \alpha_{i}, \beta_{i}(i=1-4)$, and $f_{j}(j=1-6)$ are presented in [4]. Figure 1 is a sketch on the model of the simply supported laminated composite piezoelectric rectangular thin plate under combined parametric and transverse excitations and the coordinate system.

We introduce the following transformations on (2.1):

$$
x_{1}=w_{1}, \quad x_{2}=\dot{w}_{1}, \quad x_{3}=w_{2}, \quad x_{4}=\dot{w}_{2} .
$$

Then, the following equivalent form of $(2.1)$ is obtained:

$$
\begin{aligned}
\dot{x}_{1}= & x_{2}, \\
\dot{x}_{2}= & -\omega_{1}^{2} x_{1}-\alpha_{1} x_{1} x_{3}^{2}-\alpha_{2} x_{1}^{2} x_{3}-\alpha_{3} x_{1}^{3}-\alpha_{4} x_{3}^{3}-c_{1} x_{2}+F_{1} \cos \Omega_{3} t \\
& -\left(f_{1} \cos \Omega_{1} t+f_{2} \cos \Omega_{2} t+f_{3} \cos \Omega_{4} t\right) x_{1} \\
\dot{x}_{3}= & x_{4} \\
\dot{x}_{4}= & -\omega_{2}^{2} x_{3}-\beta_{1} x_{1}^{2} x_{3}-\beta_{2} x_{1} x_{3}^{2}-\beta_{3} x_{3}^{3}-\beta_{4} x_{1}^{3}-c_{2} x_{4}+F_{2} \cos \Omega_{3} t \\
& -\left(f_{4} \cos \Omega_{1} t+f_{5} \cos \Omega_{2} t+f_{6} \cos \Omega_{4} t\right) x_{3} .
\end{aligned}
$$

The parameters of the damping and the forces in (2.3) are considered as perturbation parameters the unperturbed system is as follows:

$$
\begin{aligned}
& \dot{x}_{1}=x_{2}, \\
& \dot{x}_{2}=-\omega_{1}^{2} x_{1}-\alpha_{1} x_{1} x_{3}^{2}-\alpha_{2} x_{1}^{2} x_{3}-\alpha_{3} x_{1}^{3}-\alpha_{4} x_{3}^{3}, \\
& \dot{x}_{3}=x_{4}, \\
& \dot{x}_{4}=-\omega_{2}^{2} x_{3}-\beta_{1} x_{1}^{2} x_{3}-\beta_{2} x_{1} x_{3}^{2}-\beta_{3} x_{3}^{3}-\beta_{4} x_{1}^{3} .
\end{aligned}
$$

Executing the Maple program given by Zhang et al. [11], we can obtain the 3-order normal form of unperturbed system (2.4) as follows:

$$
\begin{aligned}
& \dot{x}_{1}=3 \omega_{1}^{4} \alpha_{3} m_{1} x_{2}^{3}+3 \omega_{1}^{2} \alpha_{3} m_{1} x_{1}^{2} x_{2}+\omega_{1}^{2} \alpha_{1} n x_{2} x_{3}^{2}+\omega_{1}^{2} \omega_{2}^{2} \alpha_{1} n x_{2} x_{4}^{2}, \\
& \dot{x}_{2}=-3 \alpha_{3} m_{1} x_{1}^{3}-3 \omega_{1}^{2} \alpha_{3} m_{1} x_{1} x_{2}^{2}-\alpha_{1} n x_{1} x_{3}^{2}-\omega_{2}^{2} \alpha_{1} n x_{1} x_{4}^{2}, \\
& \dot{x}_{3}=3 \omega_{2}^{4} \beta_{3} m_{2} x_{4}^{3}+\omega_{2}^{2} \beta_{1} n x_{1}^{2} x_{4}+\omega_{1}^{2} \omega_{2}^{2} \beta_{1} n x_{2} x_{4}^{2}+3 \omega_{2}^{2} \beta_{3} m_{2} x_{4} x_{3}^{2} \\
& \dot{x}_{4}=-3 \beta_{3} m_{2} x_{3}^{3}-\beta_{1} n x_{1}^{2} x_{3}-\omega_{1}^{2} \beta_{1} n x_{3} x_{2}^{2}-3 \omega_{2}^{2} \beta_{3} m_{2} x_{3} x_{4}^{2}
\end{aligned}
$$

where $m_{1}=1 /\left(3+2 \omega_{1}^{4}+3 \omega_{1}^{8}\right), m_{2}=1 /\left(3+2 \omega_{2}^{4}+3 \omega_{2}^{8}\right), n=1 /\left(1+\omega_{1}^{4}+\omega_{1}^{4} \omega_{2}^{4}+\omega_{2}^{8}\right)$.

Comparing (2.4) with (2.5), we can conclude that the parameters of normal form (2.5) are independent of parameters $\alpha_{2}, \alpha_{4}, \beta_{2}$, and $\beta_{4}$; that is to say, the four terms $\alpha_{2} x_{1}^{2} x_{3}, \alpha_{4} x_{3}^{3}$, $\beta_{2} x_{1} x_{3}^{2}$, and $\beta_{4} x_{1}^{3}$ in (2.4) can only have influence on the terms which are higher than cubic 


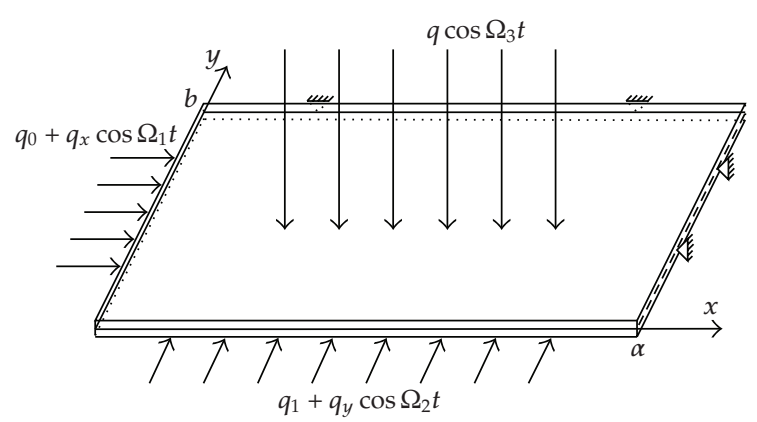

Figure 1: The model of plate subjected to its plane and transverse excitations.

of normal form (2.5). Therefore, the four terms forenamed have little contribution on 3order normal form (2.5), which means that these four nonlinear terms affect unperturbed system (2.4) to a lesser extent compared with other terms of the system. On the other hand, the analytical solution can be obtained if there are not the four terms forenamed in (2.4). With these in mind and in order to obtain the homoclinic orbits of the unperturbed system, we add the small positive parameter $\varepsilon$ on the four terms except the perturbed parameters aforementioned - the parameters of the damping and the forces.

We consider the unperturbed system (2.3), firstly, we introduce the following transformations on (2.3):

$$
x_{1}=u_{1}, \quad x_{2}=u_{2}, \quad x_{3}=v_{1}, \quad x_{4}=c_{2} v_{1}+c_{2} v_{2} .
$$

Then, the following equivalent form of (2.3) is obtained:

$$
\begin{aligned}
\dot{u}_{1}= & u_{2}, \\
\dot{u}_{2}= & -\omega_{1}^{2} u_{1}-\alpha_{1} u_{1} v_{1}^{2}-\alpha_{3} u_{1}^{3}-\alpha_{2} u_{1}^{2} v_{1}-\alpha_{4} v_{1}^{3}-c_{1} u_{2}+F_{1} \cos \Omega_{3} t \\
& -\left(f_{1} \cos \Omega_{1} t+f_{2} \cos \Omega_{2} t+f_{3} \cos \Omega_{4} t\right) u_{1}, \\
\dot{v}_{1}= & c_{2} v_{1}+c_{2} v_{2}, \\
\dot{v}_{2}= & -\bar{\omega}_{2}^{2} v_{1}-\bar{\beta}_{1} u_{1}^{2} v_{1}-\bar{\beta}_{3} v_{1}^{3}-\bar{\beta}_{2} u_{1} v_{1}^{2}-\bar{\beta}_{4} u_{1}^{3}-2 c_{2} v_{2}+\bar{F}_{2} \cos \Omega_{3} t \\
& -\left(\bar{f}_{1} \cos \Omega_{1} t+\bar{f}_{2} \cos \Omega_{2} t+\bar{f}_{3} \cos \Omega_{4} t\right) v_{1},
\end{aligned}
$$

where $\bar{\omega}_{2}^{2}=2 c_{2}+\omega_{2}^{2} / c_{2}, \bar{\beta}_{i}=\beta_{i} / c_{2}, i=1,2,3,4, \bar{f}_{j}=f_{j+3} / c_{2}, j=1,2,3, \bar{F}_{2}=F_{2} / c_{2}$.

The frequencies $\Omega_{i}(i=1,2,3,4)$ of the forces in (2.7) are rationally related, which have the following relations $Z_{1} \phi=\Omega_{1} t, Z_{2} \phi=\Omega_{2} t, \phi=\Omega_{3} t, Z_{4} \phi=\Omega_{4} t$, where $Z_{1}, Z_{2}$, and $Z_{4}$ are all nonnegative integers. Next, we add small parameter $\varepsilon$ on the four terms aforementioned 
and the parameters of the damping and the forces in (2.7). Therefore, the following system can be obtained as follows:

$$
\begin{aligned}
\dot{u}_{1}= & u_{2} \\
\dot{u}_{2}= & -\omega_{1}^{2} u_{1}-\alpha_{1} u_{1} v_{1}^{2}-\alpha_{3} u_{1}^{3}-\varepsilon \alpha_{2} u_{1}^{2} v_{1}-\varepsilon \alpha_{4} v_{1}^{3}-\varepsilon c_{1} u_{2}+\varepsilon F_{1} \cos \phi \\
& -\varepsilon\left(f_{1} \cos Z_{1} \phi+f_{2} \cos Z_{2} \phi+f_{3} \cos Z_{4} \phi\right) u_{1} \\
\dot{v}_{1}= & \varepsilon c_{2} v_{1}+\varepsilon c_{2} v_{2} \\
\dot{v}_{2}= & -\bar{\omega}_{2}^{2} v_{1}-\bar{\beta}_{1} u_{1}^{2} v_{1}-\bar{\beta}_{3} v_{1}^{3}-\varepsilon \bar{\beta}_{2} u_{1} v_{1}^{2}-\varepsilon \bar{\beta}_{4} u_{1}^{3}-2 \varepsilon c_{2} v_{2}+\varepsilon \bar{F}_{2} \cos \phi \\
& -\varepsilon\left(\bar{f}_{1} \cos Z_{1} \phi+\bar{f}_{2} \cos Z_{2} \phi+\bar{f}_{3} \cos Z_{4} \phi\right) v_{1} .
\end{aligned}
$$

Consider the cross-section of the phase space [9]

$$
\Sigma^{\phi_{0}}=\left\{\left(u_{1}, u_{2}, v_{1}, v_{2}, \phi\right) \mid \phi=\phi_{0}\right\}
$$

In the following analysis, we consider the system on the cross-section $\Sigma^{\phi_{0}}$ firstly. Then, let $\phi$ vary throughout the circle $\mathbf{S}^{1}$, see [9] for details.

Let $\varepsilon=0$ in (2.8); the new unperturbed system is of the form

$$
\begin{aligned}
& \dot{u}_{1}=u_{2}, \\
& \dot{u}_{2}=-\omega_{1}^{2} u_{1}-\alpha_{1} u_{1} v_{1}^{2}-\alpha_{3} u_{1}^{3}, \\
& \dot{v}_{1}=0, \\
& \dot{v}_{2}=-\bar{\omega}_{2}^{2} v_{1}-\bar{\beta}_{1} u_{1}^{2} v_{1}-\bar{\beta}_{3} v_{1}^{3} .
\end{aligned}
$$

The Hamiltonian for space of $u=\left(u_{1}, u_{2}\right)$ is of the form

$$
H=\frac{1}{2} \omega_{1}^{2} u_{1}^{2}+\frac{1}{2} \alpha_{1} u_{1}^{2} v_{1}^{2}+\frac{1}{4} \alpha_{3} u_{1}^{4}-\frac{1}{2} u_{2}^{2}
$$

It is noticed that system (2.10) is an uncoupled two-degree-of-freedom nonlinear system. The $v_{1}$ variable appears in $\left(u_{1}, u_{2}\right)$ components of system $(2.10)$ as a parameter since $\dot{v}_{1}=0$. We set $v_{1}=\sqrt{\bar{\omega}_{2}^{2} / \bar{\beta}_{3}}$. Consider the first two decoupled equations

$$
\begin{aligned}
& \dot{u}_{1}=u_{2}, \\
& \dot{u}_{2}=-\omega_{1}^{2} u_{1}-\alpha_{1} u_{1} v_{1}^{2}-\alpha_{3} u_{1}^{3} .
\end{aligned}
$$

Let $R=-\omega_{1}^{2}-\alpha_{1} v_{1}^{2}$, and consider $R>0, \alpha_{3}>0$. Therefore, (2.12) has three singular points: one is $\left(u_{1}, u_{2}\right)=(0,0)$, it is a saddle point, and the other two are centers: 
$\left(u_{1}, u_{2}\right)=\left( \pm \sqrt{R / \alpha_{3}}, 0\right)$; therefore, (2.12) can exhibit homoclinic bifurcations. The homoclinic orbits which connect the saddle point $\left(u_{10}, u_{20}\right)=(0,0)$ are obtained as

$$
\begin{aligned}
& u_{1}= \pm \sqrt{\frac{2 R}{\alpha_{3}}} \operatorname{sech} \sqrt{R} t \\
& u_{2}= \pm \sqrt{\frac{2}{\alpha_{3}}} R \operatorname{sech} \sqrt{R} t \tanh \sqrt{R} t .
\end{aligned}
$$

It is known from foregoing analysis that the condition $R=-\omega_{1}^{2}-\alpha_{1} v_{1}^{2}>0$ holds; in conclusion, the system considered here satisfies the following conditions:

$$
\bar{\beta}_{3} \omega_{1}^{2}<\alpha_{1} \bar{\omega}_{2}^{2}<0, \quad \alpha_{3}>0
$$

It is known from analysis in paper [9] that the first pulse Melnikov function is given by the integral

$$
M\left(v_{0}, \phi_{0}, \mu\right)=\int_{-\infty}^{+\infty}\left\langle\mathbf{n}\left(p^{h}(t)\right), \mathbf{g}\left(p^{h}(t), \omega t+\phi_{0}, \mu, 0\right)\right\rangle d t
$$

It can be seen easily that (2.8) can be written as the following form:

$$
\begin{gathered}
\dot{u}=J D_{u} H\left(u, v_{1}\right)+\varepsilon g^{u}\left(u, v, \phi_{0}, \mu, \varepsilon\right), \\
\dot{v}_{1}=\varepsilon g^{v_{1}}\left(u, v, \phi_{0}, \mu, \varepsilon\right), \\
\dot{v}_{2}=\Omega\left(u, v_{1}\right)+\varepsilon g^{v_{2}}\left(u, v, \phi_{0}, \mu, \varepsilon\right),
\end{gathered}
$$

where $u=\left(u_{1}, u_{2}\right) \in \mathbf{R}^{2}, v=\left(v_{1}, v_{2}\right) \in \mathbf{R}^{2}, \mu \in \mathbf{R}^{p}$ denotes the parameters of the system, and $J$ is a symplectic matrix

$$
J=\left(\begin{array}{rr}
0 & 1 \\
-1 & 0
\end{array}\right)
$$

Then, the vectors $\mathbf{n}$ and $\mathbf{g}$ in (2.15) are of the following form:

$$
\begin{gathered}
\mathbf{n}=\left(D_{u} H\left(u, v_{1}\right), D_{v_{1}} H\left(u, v_{1}\right)-D_{v_{1}} H\left(u_{0}\left(v_{1}\right), v_{1}\right), 0\right), \\
\mathbf{g}=\left(g^{u}(u, v, \omega t, \mu, 0), g^{v_{1}}(u, v, \omega t, \mu, 0), g^{v_{2}}(u, v, \omega t, \mu, 0)\right), \\
p^{h}(t)=\left(u^{h}\left(t, v_{1}\right), v_{1}, v_{2}^{h}\left(t, v_{1}\right)+v_{20}\right) .
\end{gathered}
$$


Mathematical Problems in Engineering

The $k$-pulse Melnikov function $M_{k}(k=1,2, \ldots)$ is defined as

$$
M_{k}\left(v_{0}, \phi_{0}, \mu\right)=\sum_{j=0}^{k-1} M\left(v_{10}, v_{20}+j \Delta v_{2}\left(v_{10}\right), \phi_{0}, \mu\right),
$$

where

$$
\Delta v_{2}\left(v_{10}\right)=\int_{-\infty}^{+\infty} \Omega\left(u^{h}\left(\tau, v_{1}\right), v_{10}\right) d \tau
$$

Based on (2.15), the 1-pulse Melnikov function is computed as follows:

$$
\begin{aligned}
M= & \int_{-\infty}^{+\infty} u_{2}\left[F_{1} \cos \phi-\alpha_{2} u_{1}^{2} v_{1}-\alpha_{4} v_{1}^{3}-c_{1} u_{2}-\left(f_{1} \cos Z_{1} \phi+f_{2} \cos Z_{2} \phi+f_{3} \cos Z_{4} \phi\right) u_{1}\right] d t \\
& -c_{2}\left(v_{1}+v_{2}\right)\left(-\bar{\omega}_{2}^{2} v_{1}-\bar{\beta}_{1} u_{1}^{2} v_{1}-\bar{\beta}_{3} v_{1}^{3}\right) d t \\
= & \sum_{i=1,2,4} \frac{f_{i} \Omega_{i}^{2} \pi}{\alpha_{3}} \sin \left(Z_{i} \phi_{0}\right) \operatorname{csch} \frac{\Omega_{i} \pi}{2 \sqrt{R}}-\frac{4 c_{1} R^{3 / 2}}{3 \alpha_{3}}-F_{1} \pi \sqrt{\frac{2}{\alpha_{3}}} \sin \phi_{0} \operatorname{sech} \frac{\pi}{2 \sqrt{R}}-c_{2} \Delta v_{2}\left(v_{10}+v_{20}\right)
\end{aligned}
$$

where

$$
\Delta v_{2}=\int_{-\infty}^{+\infty}\left(-\bar{\omega}_{2}^{2} v_{1}-\bar{\beta}_{1} u_{1}^{2} v_{1}-\bar{\beta}_{3} v_{1}^{3}\right) d t=-\frac{\alpha_{1}}{\alpha_{3}} \sqrt{\frac{\bar{\omega}_{2}^{2} R}{-\bar{\beta}_{3}}}
$$

Based on (2.19) and the foregoing analysis, the $k$-pulse Melnikov function is obtained as follows:

$$
\begin{aligned}
M_{k}= & \sum_{i=1,2,4} \frac{f_{i} \Omega_{i}^{2} \pi}{\alpha_{3}} k \sin \left(Z_{i} \phi_{0}\right) \operatorname{csch} \frac{\Omega_{i} \pi}{2 \sqrt{R}}-\frac{4 c_{1} R^{3 / 2}}{3 \alpha_{3}} k-F_{1} \pi k \sqrt{\frac{2}{\alpha_{3}}} \sin \phi_{0} \operatorname{sech} \frac{\pi}{2 \sqrt{R}} \\
& -c_{2} \Delta v_{2} v_{10} k-c_{2} \Delta v_{2} v_{20} k-\frac{k(k-1)}{2} c_{2} \Delta v_{2}^{2} .
\end{aligned}
$$

If the $k$-pulse Melnikov function $M_{k}$ has simple zero points, then we obtain

$$
\begin{aligned}
& \sum_{i=1,2,4} \frac{f_{i} \Omega_{i}^{2} \pi}{\alpha_{3}} \sin \left(Z_{i} \phi_{0}\right) \operatorname{csch} \frac{\Omega_{i} \pi}{2 \sqrt{R}}-\frac{4 c_{1} R^{3 / 2}}{3 \alpha_{3}}-F_{1} \pi \sqrt{\frac{2}{\alpha_{3}}} \sin \phi_{0} \operatorname{sech} \frac{\pi}{2 \sqrt{R}} \\
&-c_{2} \Delta v_{2} v_{10}-c_{2} \Delta v_{2} v_{20}-\frac{(k-1)}{2} c_{2} \Delta v_{2}^{2}=0 .
\end{aligned}
$$


It is known that the following holds:

$$
D_{v_{10}} M_{k}=-c_{2} \Delta v_{2} k=\frac{c_{2} k \alpha_{1}}{\alpha_{3}} \sqrt{\frac{\bar{\omega}_{2}^{2} R}{-\bar{\beta}_{3}}} \neq 0
$$

Equation (2.24) can be rewritten as follows:

$$
\begin{aligned}
k= & \sum_{i=1,2,4} \frac{2 f_{i} \Omega_{i}^{2} \pi}{\alpha_{3} c_{2} \Delta v_{2}^{2}} \sin \left(Z_{i} \phi_{0}\right) \operatorname{csch} \frac{\Omega_{i} \pi}{2 \sqrt{R}}-\frac{8 c_{1} R^{3 / 2}}{3 \alpha_{3} c_{2} \Delta v_{2}^{2}}-\frac{2 F_{1} \pi}{c_{2} \Delta v_{2}^{2}} \sqrt{\frac{2}{\alpha_{3}}} \sin \phi_{0} \operatorname{sech} \frac{\pi}{2 \sqrt{R}} \\
& -\frac{2 c_{2}\left(v_{10}+v_{20}\right)}{\Delta v_{2}}+1 .
\end{aligned}
$$

For $k \in \mathbf{N}$, we may choose proper parameters in (2.26) so that the value of the following formula:

$$
\sum_{i=1,2,4} \frac{2 f_{i} \Omega_{i}^{2} \pi}{\alpha_{3} c_{2} \Delta v_{2}^{2}} \sin \left(Z_{i} \phi_{0}\right) \operatorname{csch} \frac{\Omega_{i} \pi}{2 \sqrt{R}}-\frac{8 c_{1} R^{3 / 2}}{3 \alpha_{3} c_{2} \Delta v_{2}^{2}}-\frac{2 F_{1} \pi}{c_{2} \Delta v_{2}^{2}} \sqrt{\frac{2}{\alpha_{3}}} \sin \phi_{0} \operatorname{sech} \frac{\pi}{2 \sqrt{R}}-\frac{2 c_{2}\left(v_{10}+v_{20}\right)}{\Delta v_{2}}
$$

is a nonnegative integer. Based on analysis in [9], the stable manifold $W^{s}\left(M_{\varepsilon}^{\phi_{0}}\right)$ and unstable manifold $W^{u}\left(M_{\varepsilon}^{\phi_{0}}\right)$ of system (2.8) intersect transversely, which means that the multipulse chaotic motions exist in the simply supported laminated composite piezoelectric rectangular thin plate. To verify the analytical results obtained above, numerical method will be utilized to indicate that the multipulse chaotic motions occur for the laminated composite piezoelectric plate.

\section{Numerical Simulations}

In this section, the nonlinear dynamic behaviors of the simply supported laminated composite piezoelectric rectangular thin plate under combined parametric and transverse excitations are presented by using Matlab programs.

We choose parameters of (2.3) under condition (2.14) to do numerical simulations. The parameters are chosen as follows: $\omega_{1}=5.6, \alpha_{1}=2.8, \alpha_{2}=-2.5, \alpha_{3}=10.2, \alpha_{4}=1.8, \mu_{1}=0.18$, $f_{1}=10, f_{2}=12, f_{3}=20, F_{1}=10, \Omega_{1}=1, \Omega_{2}=2, \Omega_{4}=1.2, \omega_{2}=6.1, \beta_{1}=2.2, \beta_{2}=8.3, \beta_{3}=-3$, $\beta_{4}=2.4, \mu_{2}=0.19, f_{4}=12, f_{5}=11, f_{6}=28, F_{2}=2, \Omega_{3}=\phi=1$. The initial values are given by Matlab stochastically as follows: $x_{10}=-0.1465, x_{20}=0.1909, x_{30}=0.1892, x_{40}=-0.0376$. Figure 2 demonstrates chaotic motions of the laminated composite piezoelectric plate. Figures 2(a) and 2(c) present the waveform on the planes $\left(t, x_{1}\right)$ and $\left(t, x_{3}\right)$. Figures 2(b) and 2(d) give the phase portraits on the planes $\left(x_{1}, x_{2}\right)$ and $\left(x_{3}, x_{4}\right)$. Multipulse jumping motions of system (2.3) can be obtained apparently from phrase portraits of Figures 2(e) and 2(f) in three-dimensional spaces $\left(x_{1}, x_{2}, x_{3}\right)$ and $\left(x_{3}, x_{4}, x_{1}\right)$.

The largest Lyapunov exponents are calculated for the laminated composite piezoelectric plate. Figure 3 represents the largest Lyapunov exponents of (2.3) with variations of 


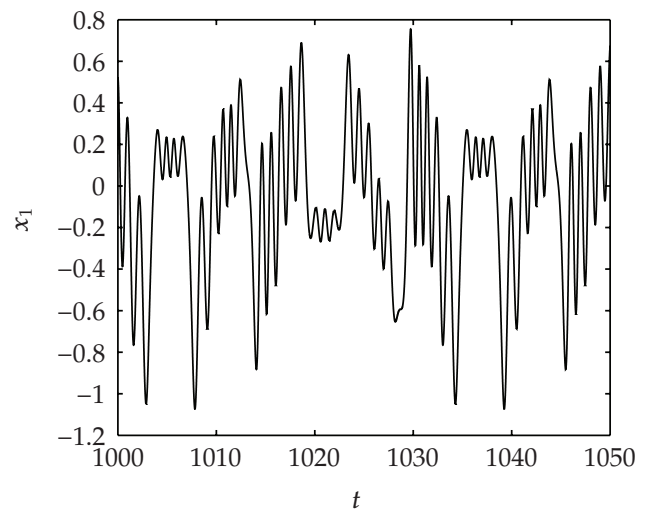

(a)

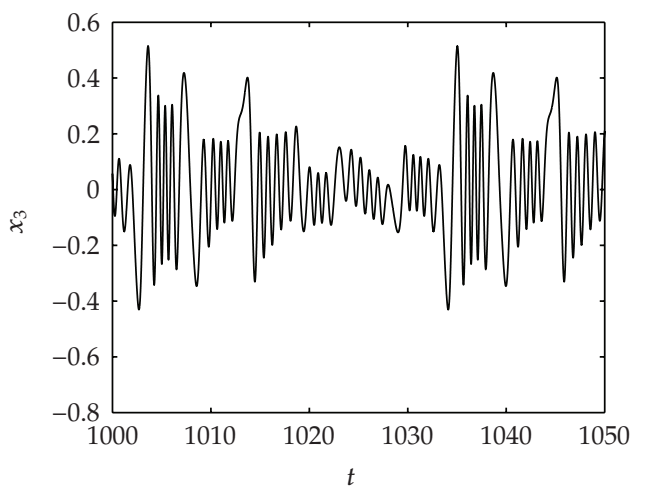

(c)

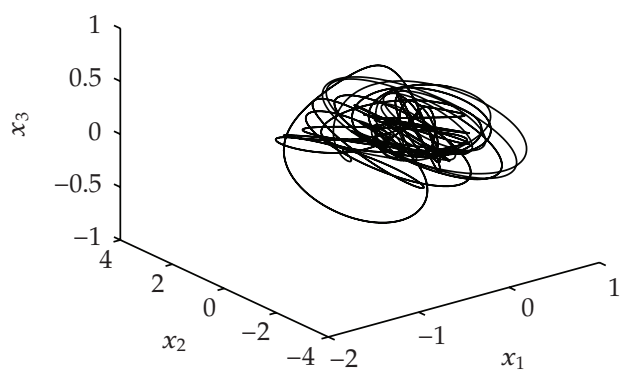

(e)

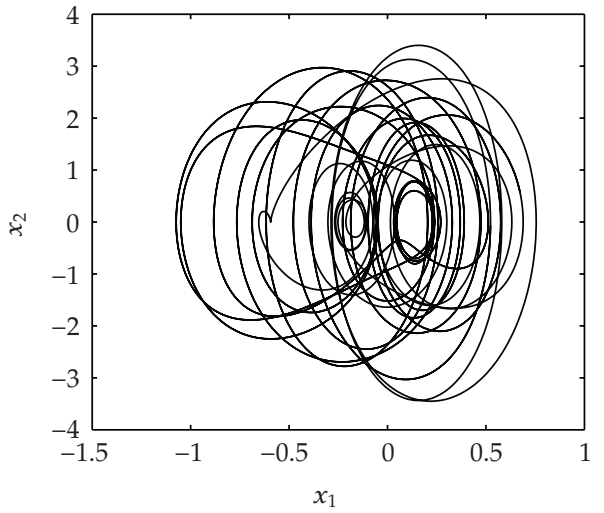

(b)

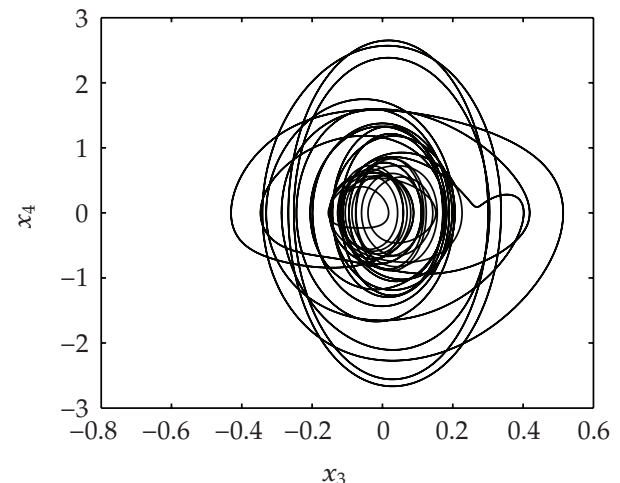

(d)

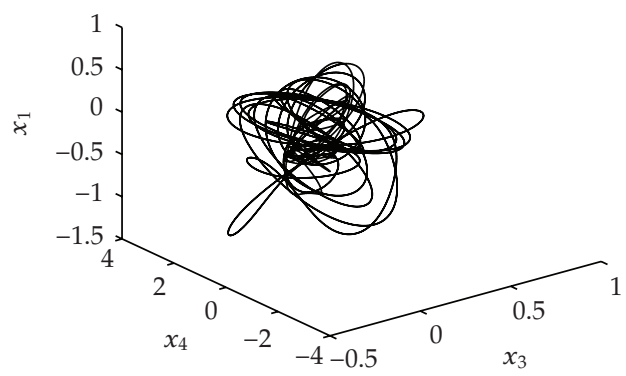

(f)

Figure 2: Chaotic motions of the laminated composite piezoelectric plate.

parametric excitation $f_{3}$ which dependent on piezoelectric excitation. Figure 4 is the largest Lyapunov exponents via frequency $\Omega_{3}=\phi$ of external force.

\section{Conclusions}

New and valuable results of analysis and computation have been achieved during the course of present study. According to our analysis, the extended Melnikov method is attributed to 


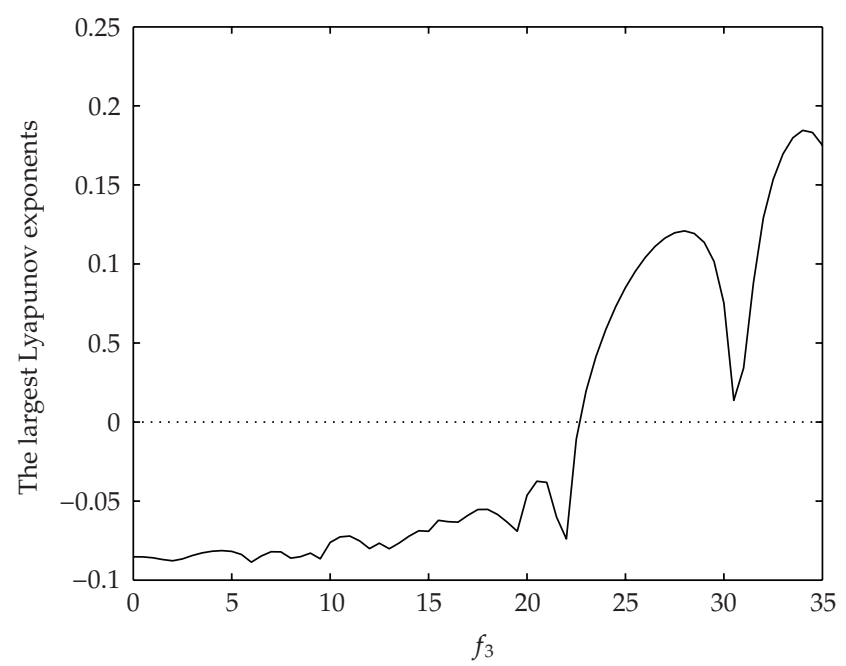

Figure 3: The largest Lyapunov exponents via $f_{3}$.

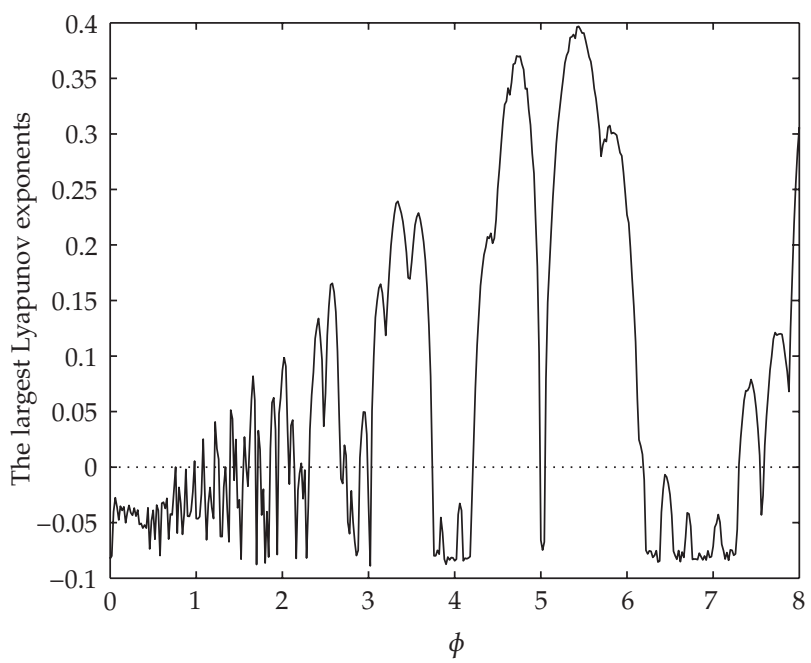

Figure 4: The largest Lyapunov exponents via $\phi$.

the nonautonomous ordinary differential equations of motion for the laminated composite piezoelectric rectangular plate by introducing the cross-section $\Sigma^{\phi_{0}}$.

We minimize the simplification processes on the system at the best possibility so that a better understanding of the nature and behavior of high-dimensional nonlinear systems can be acquired. By virtue of the theory of normal form, some nonlinear terms in the governing equations of the laminated composite piezoelectric rectangular plate have less effect than other terms on the system. Therefore, these nonlinear terms are retained herein and added with small positive parameters to analyze the complex nonlinear dynamics of the laminated composite piezoelectric rectangular plate. We obtain the simple zero point of the $k$-pulse Melnikov function, which means that there exist $k$-pulse chaotic motions for the laminated composite piezoelectric rectangular plate. The chaotic dynamics are 
also numerically investigated by means of the phase portraits and the largest Lyapunov exponents.

\section{Acknowledgments}

The authors gratefully acknowledge the support of the National Natural Science Foundation of China (NNSFC) through Grant no. 10732020, 11072008, and 11002005 and the Funding Project for Academic Human Resources Development in Institutions of Higher Learning under the Jurisdiction of Beijing Municipality (PHRIHLB).

\section{References}

[1] S. C. Her and C. S. Lin, "Deflection of cross-ply composite laminates induced by piezoelectric actuators," Sensors, vol. 10, no. 1, pp. 719-733, 2010.

[2] R. Ye and H. S. Tzou, "Control of adaptive shells with thermal and mechanical excitations," Journal of Sound and Vibration, vol. 231, no. 5, pp. 1321-1338, 2000.

[3] H. S. Shen, "Nonlinear bending analysis of unsymmetric cross-ply laminated plates with piezoelectric actuators in thermal environments," Composite Structures, vol. 63, no. 2, pp. 167-177, 2004.

[4] W. Zhang, Z. Yao, and M. Yao, "Periodic and chaotic dynamics of composite laminated piezoelectric rectangular plate with one-to-two internal resonance," Science in China, Series E, vol. 52, no. 3, pp. 731-742, 2009.

[5] M. Tanveer and A. V. Singh, "Nonlinear forced vibrations of laminated piezoelectric plates," Journal of Vibration and Acoustics, Transactions of the ASME, vol. 132, no. 2, Article ID 021005, pp. 1-13, 2010.

[6] D. A. F. Torres and P. D. T. R. Mendonca, "HSDT-layerwise analytical solution for rectangular piezoelectric laminated plates," Composite Structures, vol. 92, no. 8, pp. 1763-1774, 2010.

[7] G. Kovacic and S. Wiggins, "Orbits homoclinic to resonances, with an application to chaos in a model of the forced and damped sine-Gordon equation," Physica D, vol. 57, no. 1-2, pp. 185-225, 1992.

[8] R. Camassa, G. Kovacic, and S. K. Tin, "A Melnikov method for homoclinic orbits with many pulses," Archive for Rational Mechanics and Analysis, vol. 143, no. 2, pp. 105-193, 1998.

[9] W. Zhang, J. H. Zhang, and M. H. Yao, "The extended Melnikov method for non-autonomous nonlinear dynamical systems and application to multi-pulse chaotic dynamics of a buckled thin plate," Nonlinear Analysis, vol. 11, no. 3, pp. 1442-1457, 2010.

[10] W. Zhang, J. H. Zhang, M. H. Yao, and Z. G. Yao, "Multi-pulse chaotic dynamics of non-autonomous nonlinear system for a laminated composite piezoelectric rectangular plate," Acta Mechanica, vol. 211, no. 1-2, pp. 23-47, 2010.

[11] W. Zhang, F. X. Wang, and J. W. Zu, “Computation of normal forms for high dimensional nonlinear systems and application to nonplanar motions of a cantilever beam," Journal of Sound and Vibration, vol. 278, pp. 949-974, 2004. 


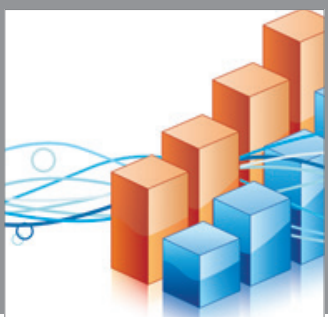

Advances in

Operations Research

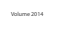

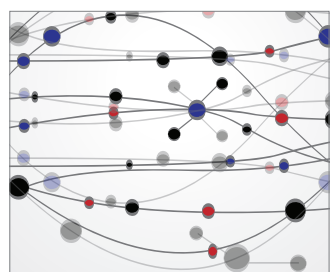

\section{The Scientific} World Journal
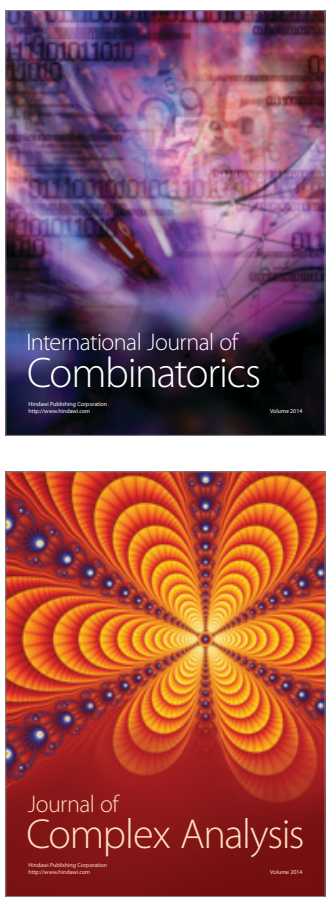

International Journal of

Mathematics and

Mathematical

Sciences
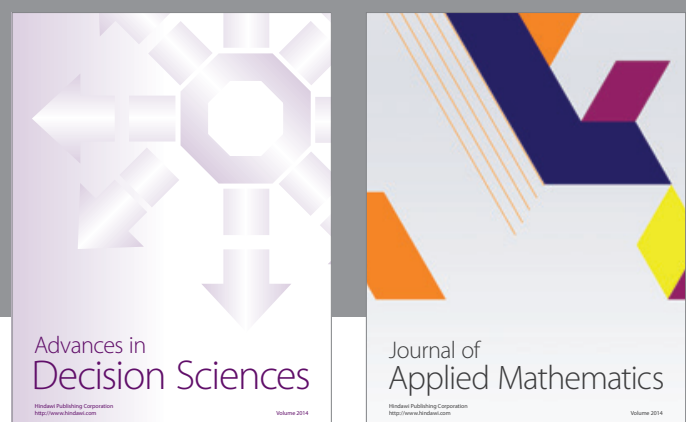

Journal of

Applied Mathematics
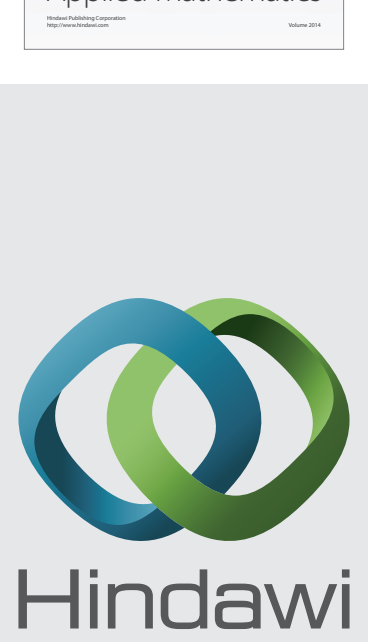

Submit your manuscripts at http://www.hindawi.com
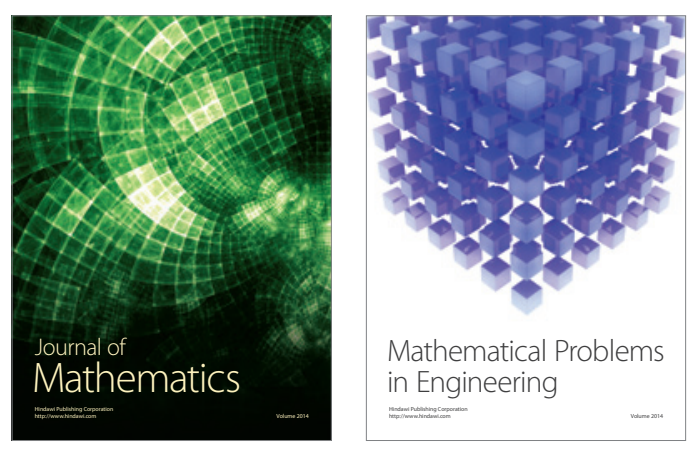

Mathematical Problems in Engineering
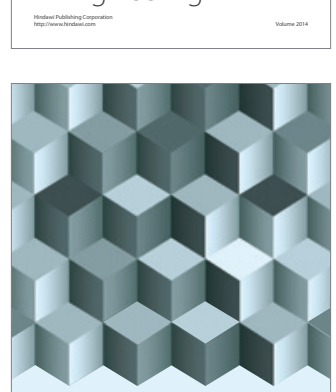

Journal of

Function Spaces
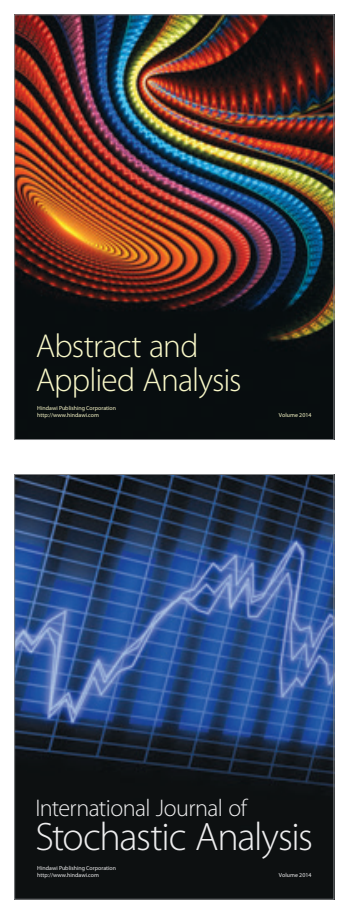

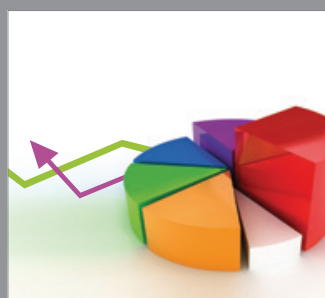

ournal of

Probability and Statistics

Promensencen
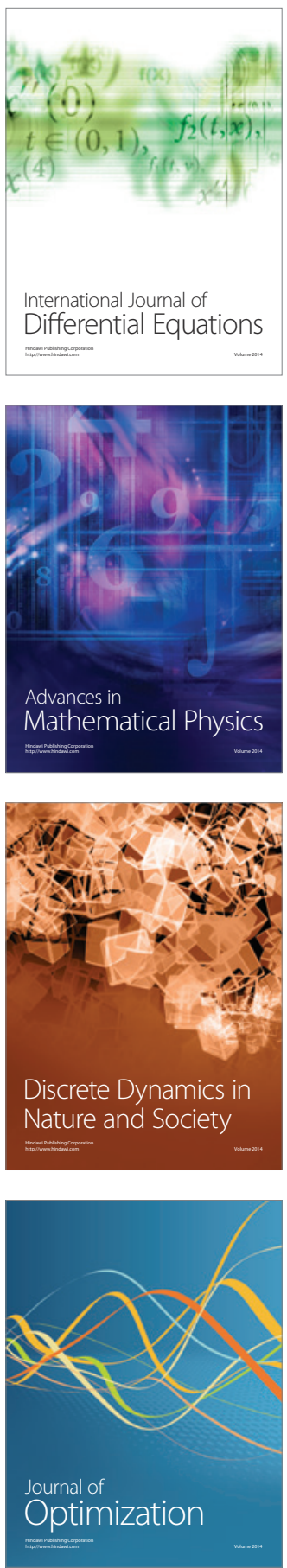